CERN-PPE/95-110

CERN-LAA/SF/95-14

16 June 1995

\title{
AN ISPA-CAMERA FOR GAMMA RAYS
}

\author{
D. Puertolas a, D. Piedigrossia ${ }^{a}$ R. Pani, H. Leutz ${ }^{a}$, \\ T. Gys a , F. de Notaristefanic ${ }^{\mathrm{c}}$, C. D’Ambrosio ${ }^{\mathrm{a}}$ \\ a CERN, Geneva, Switzerland \\ b Dept of Experimental Medicine, University of Rome "La Sapienza", Italy \\ c INFN Section of Rome and 3rd University of Rome, Italy
}

\begin{abstract}
With the recently developed ISPA (Imaging Silicon Pixel Array)-tube attached either to a planar $\mathrm{YAlO}_{3}(\mathrm{Ce})$ (YAP) disc $(1 \mathrm{~mm}$ thick) or to a matrix of optically-separated YAP-crystals (5mm high, $0.6 \times 0.6 \mathrm{~mm}^{2}$ cross-section) we achieved high spatial resolution of ${ }^{57} \mathrm{Co}-122 \mathrm{keV}$ photons. The vacuum-sealed ISPA-tube is only $4 \mathrm{~cm}$ long with $3.5 \mathrm{~cm}$ diameter and consists of a photocathode viewed at $3 \mathrm{~cm}$ distance by a silicon pixel chip, directly detecting the photoelectrons. The chip-anode consists of 1024 rectangular pixels with $75 \mu \mathrm{m} \times 500 \mu \mathrm{m}$ edges, each bump-bonded to their individual front-end electronics. The total pixel array read-out time is $10 \mu \mathrm{s}$. The measured intrinsic spatial resolutions (FWHM) of this ISPA-camera are $700 \mu \mathrm{m}$ (planar YAP) and $310 \mu \mathrm{m}$ (YAP-matrix). Apart from its already demonstrated application for particle tracking with scintillating fibres, the ISPA-tube provides also an excellent tool in medicine, biology and chemistry.
\end{abstract}

Submitted to IEEE Transactions on Nuclear Science 


\section{INTRODUCTION}

In addition to its applications in particle physics [1,2] and for photon counting of weak light sources [3], we have now also employed the Imaging Silicon Pixel Array (ISPA)-tube for position-sensitive light detection in a $\gamma$-camera. The aim is finally to locate radio-activities in tissues on a submillimetre scale for medical diagnostics.

$\gamma$-cameras for medical applications consist essentially of an image-producing collimator and a detector, which converts it into a visible picture. This development started in the fifties [4] with the scintillation cameras of H. O. Anger, which are still in use and achieve now-a-days spatial resolutions of $3 \mathrm{~mm}$ (FWHM) [5] with $12 \mathrm{~mm}$ thick planar $\mathrm{NaI}(\mathrm{Tl})$ crystals of up to $50 \mathrm{~cm}$ diameter viewed by an array of photomultipliers.

More recent developments are based on position-sensitive photomultipliers (PSPM) (Hamamatsu R2486 [6]). H. Uchida et al. [7] coupled an array of closely packed BGO-crystals with $5 \times 5 \mathrm{~mm}^{2}$ cross-section and $20 \mathrm{~mm}$ height to the photocathode of an R2486-tube. They obtained with $511 \mathrm{keV}$ annihilation coincidences $3.2 \mathrm{~mm}$ (FWHM) spatial resolution. J. M. Poulsen et al. [8] applied a $3 \mathrm{~mm}$ thick $\mathrm{NaI}(\mathrm{Tl})$ planar disc on a R2486-tube and achieved $1.7 \mathrm{~mm}$ (FWHM) spatial resolution at $122 \mathrm{keV} \gamma$-energy. With a planar $5 \mathrm{~mm}$ thick $\mathrm{Gd}_{2} \mathrm{SiO}_{5}(\mathrm{Ce})$-crystal Y. Nagai and $\mathrm{T}$. Hyodo [9] measured with an R2486-tube $2.2 \mathrm{~mm}$ (FWHM) spatial resolution at $511 \mathrm{keV} \gamma$-energy. With an array of $\mathrm{YAlO}_{3}(\mathrm{Ce})$-crystals (YAP) $\left(0.6\right.$ x $0.6 \mathrm{~mm}{ }^{2}$ crosssection and $7 \mathrm{~mm}$ height), again mounted on an R2486-tube, R. Pani et al. [10] arrived finally at a best value of $0.7 \mathrm{~mm}$ (FWHM) intrinsic spatial resolution at $140 \mathrm{keV} \gamma$ energy.

Double-sided silicon strips represent a different class of detectors for the soft Xray region $(10-100 \mathrm{keV})[11,12]$. The silicon readout pitch is $0.1 \mathrm{~mm}$ with capacitive charge division by means of floating strips in between. With masks containing parallel slits of increasing spatial frequencies, an X-ray separation of $0.2 \mathrm{~mm}$ was achieved [12] at $60 \%$ to $70 \%$ of the Contrast Transfer Function. However, such detectors suffer mainly from the low $\gamma$-detection efficiency of silicon : at $25 \mathrm{keV}$ they provide $14 \%$ total $\gamma$-absorption efficiency; this number drops rapidly to $2.1 \%(60 \mathrm{keV})$ and $1.4 \%$ $(90 \mathrm{keV})$ where most of the attenuation is Compton-scattering. This poor detection efficiency is expected to improve through the development of pixel structures in GaAs.

In the following, results from measurements with a $\gamma$-camera employing a novel type of light detector are reported. The scintillations emitted from a $\gamma$-converting $1 \mathrm{~mm}$ thick planar YAP-disc or from a matrix of optically-separated YAP-crystals are in our arrangement detected with an ISPA-tube. 


\section{THE ISPA-TUBE}

Instead of a phosphor screen as with conventional image intensifiers, the ISPAtube ${ }^{\# 1}$ contains a silicon chip anode, subdivided into small pixels, which are bombarded with electrons emitted from a light-detecting photocathode. Each electron hitting the silicon creates 280 electron-hole pairs per $\mathrm{kV}$ acceleration. With $25 \mathrm{kV}$ potential difference between photocathode and chip anode, the total amount of electron-hole pairs is around 7000, a number which is about three orders of magnitude higher than the electron multiplication at the first dynode of a photomultiplier. This high gain along with the Fano factor ( 0.1 in silicon) results in correspondingly much smaller statistical fluctuations.

Physically, the ISPA-tube represents a vacuum-sealed cylinder of $40 \mathrm{~mm}$ length and $35 \mathrm{~mm}$ diameter (Fig. 1). A $18 \mathrm{~mm}$ diameter photocathode, evaporated onto the inside surface of an optical fibre window for collimated light passage, is viewed at $30 \mathrm{~mm}$ distance by an $8 \mathrm{~mm} \times 4.8 \mathrm{~mm}$-area silicon chip ${ }^{\# 2}$. It contains 1024 pixels of $75 \mu \mathrm{m} \times 500 \mu \mathrm{m}$ size. Each detector pixel is bump-bonded to an equally-sized pixel front-end electronics, which comprises preamplifier, comparator with adjustable threshold, delay line, coincidence logic and memory element [13,14]. The pixel response is thus binary and the bump-bonded individual electronics minimizes the noise. Its strobed (gated) output allows self-triggering (via the analog back-contact signal of the detector chip) or external triggering. The line-parallel readout ${ }^{\# 3}$ of the pixel array reduces the readout time to $10 \mu$ s and the connections leading through the vacuum-sealed envelope are only 30 instead of more than a thousand.

\section{EXPERIMENTAL ARRANGEMENT}

Our camera set-up is shown in Fig. 1. It consists of a ${ }^{57} \mathrm{Co}$-source (principal $\gamma$ emission at $122 \mathrm{keV}$ ) and a $5 \mathrm{~mm}$ thick lead collimator (phantom) with five holes of $0.25 \mathrm{~mm}$ diameter each. The positions of the holes are indicated in the insert of Fig. 1. The two main components of the camera are the crystal for $\gamma$-conversion and the ISPAtube for position-sensitive detection of the crystal-scintillations.

The $122 \mathrm{keV}$ gammas (replacing the $140 \mathrm{keV}$ gammas of $99 \mathrm{~m} \mathrm{Tc}$ ( $6 \mathrm{~h}$ half-life) applied in mammography) emitted from the ${ }^{57} \mathrm{Co}$-source were attenuated in two different crystal configurations mounted alternately in front of the ISPA fibre-optics input window. The first configuration was a $1 \mathrm{~mm}$ thick $(9 \mathrm{~mm}$ diameter) planar YAP(Ce)-disc which converts $14 \%$ of these gammas by photoeffect and $\sim 7 \%$ by

\footnotetext{
\#1 Assembled and manufactured by BV Delft Electronische Producten (DEP), NL-9300 AB Roden, The Netherlands. The chip was wire bonded at U.C.I. Microélectronique, F-91946 Les Ulis, France.

\#2 The chip was developed by the ECP microelectronics group and the RD19 Collaboration at CERN.

\#3 The 64 columns of 16 pixels can be readout at a frequency of 5 to $10 \mathrm{MHz}$, resulting in readout times of 6 to $12 \mu \mathrm{s}$.
} 
Compton scattering. The second configuration was a crystal array composed of YAP $(\mathrm{Ce})$ scintillating elements ${ }^{\# 4}$, with $0.6 \times 0.6 \mathrm{~mm}^{2}$ cross-section each and $5 \mathrm{~mm}$ thickness. The photo-absorption and Compton scattering fractions are $53 \%$ and $30 \%$, respectively. The main properties of YAP-crystals are listed in table 1. The YAP-light emission overlaps with about $15 \%$ the spectral sensitivity of the present ISPAphotocathode, resulting in an average quantum efficiency of only $3 \%$. This overlap would considerably increase if the ISPA-tube was equipped with a quartz window. The ISPA-electronics time response is well adapted to the YAP light decay of $27 \mathrm{~ns}$.

The principle of gamma absorption with subsequent photon conversion and photoelectron detection is illustrated in Fig. 2. The YAP-scintillation light is transmitted through the optical window of the ISPA-tube to its photocathode. There, it is converted into photoelectrons which are accelerated towards the silicon detector chip. For this particular application we used the ISPA-tube in the proximity-focussed mode.

In order to trigger only on $\gamma$-conversions, we installed a self-triggering system (Fig. 3) : fast analog signals from the bias contact of the detector chip, arising from the photoelectrons generated by each gamma absorption, were appropriately amplified and shaped and finally transmitted to a lower- and an upper-level discriminator. This energy window activates the strobed signal output of the ISPA-tube and starts the pixel array readout. All signals falling outside this window are discarded.

\section{MEASUREMENTS AND RESULTS}

Figures $4 \mathrm{a}$ and $4 \mathrm{~b}$, display pulse height distributions of the analog signals arising from the bias contact of the ISPA-tube detector chip. They represent the energy spectra of ${ }^{57} \mathrm{Co}$ measured in the same conditions for the YAP disc and array, respectively. Light reduction from the disc to the array can be explained by the array characteristics [10]. As mentioned in the preceeding paragraph, we used these analog signals as a selftrigger. An important feature for the data acquisition is the setting of the two discriminator levels which form the energy window for the strobe activation. Due to the excellent visibility of the photoelectron peaks, an inherent characteristic of our ISPAtube, the energy scale can be readily calibrated into photoelectrons $[15,16]$. The lower level discriminator was set to exclude photoelectron numbers below 3 for both the disc and the array detectors, in order to provide a clear signal-to-noise ratio. The upper level discriminator was set to 25 photoelectrons. For each accepted gamma event, the pattern produced on the pixel array is recorded and its center-of-gravity calculated, together with its precision. These values represent the gamma position and its error, respectively. The accumulation of the centers of gravity finally forms the gamma image of the phantom. The distribution of the pixel residuals ${ }^{\# 5}$ corresponds to the average light spread on the photocathode for each gamma event. In the case of the plate, the shape of

\footnotetext{
\#4 The YAP-crystal array was produced by Preciosa Crytur Ltd, Turnov, Czech Republic.

\#5 Relative coordinates of each pixel with respect to the center-of-gravity.
} 
the measured light spread is Lorentzian\#, with a FWHM of $1.2 \mathrm{~mm}$, while it is Gaussian with FWHM of $0.65 \mathrm{~mm}$ for the array.

Two-dimensional views of $122 \mathrm{keV}$ gamma exposures are shown in figures $5 \mathrm{a}$ (YAP planar disc) and 5b (YAP-array). The five holes of the lead collimator (see insert of Fig. 1) are clearly visible. The intensity variations between the holes are due to the spatial non-uniformity of the gamma source. The small background level is attributed to scatterings along the lead collimator and within the YAP detectors. Figures $6 \mathrm{a}$ and $6 \mathrm{~b}$ show the corresponding three-dimensional pictures and for both cases, the excellent contrast.

Figures $7 \mathrm{a}$ and $7 \mathrm{~b}$ display the intensity profile taken along the lines $\mathrm{A}$ indicated in figures $5 \mathrm{a}$ and $5 \mathrm{~b}$. The peaks are nearly Gaussian with a FWHM of $700 \mu \mathrm{m}$ and 310 $\mu \mathrm{m}$, respectively (intrinsic spatial resolutions). Finally, figure 8 shows the precision on the centre-of-gravity positions for the planar YAP and the matrix YAP.

\section{DISCUSSION}

There is a fundamental difference between the YAP-plate and the YAP-array. The first represents a continuous medium, whereas the second one is a discrete sampling device. With the plate, the calculated total spatial resolution is given by :

$$
(\mathrm{FWHM})_{\text {tot }}^{\text {plate }}=2.36 \sigma=2.36 \frac{0.9}{\sqrt{8.8}}=0.71 \mathrm{~mm}
$$

where $0.9 \mathrm{~mm}$ is the measured standard deviation of the residuals, and 8.8 is the average photoelectron number of the spectrum shown in figure $4 \mathrm{a}$. The value of 0.71 $\mathrm{mm}$ is in good agreement with the measured $0.7 \mathrm{~mm}$ (Fig. 7a).

With the array, the calculated total spatial resolution is :

$$
(\mathrm{FWHM})_{\text {tot }}^{\text {array }}=\sqrt{0.6^{2}+\left(\frac{2.36 \times 0.28}{\sqrt{6.4}}\right)^{2}}=\sqrt{0.6^{2}+0.26^{2}}=0.65 \mathrm{~mm}
$$

where the first term $\left(0.6^{2}\right)$ is the crystal size (sampling term) and the second $\left(0.26^{2}\right)$ is the intrinsic spatial resolution. The value of $0.28 \mathrm{~mm}$ is the measured standard deviation of the residuals and 6.4 is the average photoelectron number of the spectrum shown in figure $4 \mathrm{~b}$. The statistical estimation of $0.26 \mathrm{~mm}$ is to be compared with the measured $0.31 \mathrm{~mm}$ (Fig. 7b). From relations (1) and (2), it can be seen that the resolution, in the case of the plate, is limited by the light spread on the photocathode and by the actual average number of detected photoelectrons per event. On the contrary, in the array case, the limiting factor is given by the crystal size $(0.6 \mathrm{~mm})$, while the intrinsic resolution is as small as $0.26 \mathrm{~mm}$. It is again worth noting that with a better

\footnotetext{
\#6 Assuming the scintillation light emitted isotropically, the intensity distribution on the input window results from a spherical wave intercepted by a plane. This corresponds to a Lorentzian shape.
} 
matched photocathode, the number of detectable photoelectrons can be increased by a factor 2 to 4, improving in both cases space and energy resolutions.

The excellent intrinsic resolution is given by the inherent response of the ISPAtube. This becomes even clearer if we compare relation (2) with the relation obtained with a PSPM-tube by Pani et al. [10] (who applied the same YAP-array) :

$$
(\mathrm{FWHM})_{\mathrm{tot}}=\sqrt{0.6^{2}+\left(\frac{8.0}{\sqrt{80}}\right)^{2}}=\sqrt{0.6^{2}+0.9^{2}}=1.08 \mathrm{~mm}
$$

where the first term is again the crystal size (sampling term) and the second is the intrinsic spatial resolution of the PSPM-tube. The value of $8.0 \mathrm{~mm}$ is the FWHM of the residuals and 80 the average photoelectron number. Our estimation of $1.08 \mathrm{~mm}$ is to be compared with the value of $1.0 \mathrm{~mm}$ quoted in [10]. As can be seen, the striking difference between the two cases is that in relation (3) the resolution is dominated by the charge spread of the PSPM-tube. Even when the total number of photoelectrons is 80 (compared with 6.4 in our case), the intrinsic resolution of the PSPM is still $0.9 \mathrm{~mm}$, compared with $0.26 \mathrm{~mm}$ for our ISPA-tube.

\section{CONCLUSIONS}

Comparing our spatial resolution with those [7 to 10] obtained by applying position-sensitive photomultipliers [6], it becomes obvious that the improvement is due to the light detection with the ISPA-tube. The total spatial resolution at FWHM is $0.7 \mathrm{~mm}$ for the plate and the intrinsic spatial resolution at FWHM is $0.31 \mathrm{~mm}$ for the array. The detection efficiencies are dictated by the crystal thicknesses, and estimated to be $20 \%$ and $70 \%$ at $122 \mathrm{keV}$, after the cuts below three photoelectrons (Figs 4a and $4 b)$.

The next step of improvements will be to enlarge the ISPA-anode by arranging four silicon chips close to each other in the same ISPA-envelope. Since the spatial resolution of the ISPA-tube is better than the one given by the YAP-elements, it is possible to demagnify the ISPA picture between three and five times without loss in overall resolution. This can be done by an appropriate electrostatic focussing between photocathode and pixel-anode of the ISPA-tube. In this case, one ISPA-tube would cover a crystal area of up to $80 \times 80 \mathrm{~mm}^{2}$.

\section{ACKNOWLEDGEMENTS}

We are indebted to A. Zichichi for supporting our work. We thank E. Heijne and M. Campbell for their advice as well as F. Cindolo and A. Menchikov for their help in software and hardware. Finally, we acknowledge the efficient work of our industrial partners. 


\section{REFERENCES}

[1] T. Gys et al., "A new position-sensitive photon detector based on an imaging silicon pixel array (ISPA-tube)", Nucl. Instr. and Meth. in Physics Res., vol. A355, 1995, p. 386.

[2] C. D'Ambrosio et al., "First beam exposures of a scintillating fibre tracker readout by an ISPA-tube“, Nucl. Instr. and Meth. in Physics Res., vol. A359, 1995, p. 618.

[3] C. D'Ambrosio et al., "Position-sensitive photon counting with an ISPA-tube", I.E.E.E. Trans. Nucl. Sci. , vol. 42, 1995, p. 130.

[4] H.O. Anger, "Scintillation camera“, Rev. Sci. Instr., vol. 29, 1958, p. 27.

H.O. Anger, "Sensitivity, resolution and linearity of the scintillation camera", IEEE Trans. Nucl. Sci., NS-13, 1966, p. 380.

[5] M.T. Madsen et al., Phys. Med. Biol., vol. 37, 1992, p. 1625.

[6] H. Kume et al., "Position sensitive photomultiplier tubes for scintillation imaging“, IEEE Trans. Nucl. Sci., NS-33, 1986, p. 359.

[7] H. Uchida et al., "Design of a mosaic BGO detector system for positron CT", IEEE Trans. Nucl. Sci., NS-33, 1986, p. 464.

[8] J.M. Poulsen et al., "Position sensitive scintillation detector for hard X-rays", Nucl. Instr. and Meth. in Physics Res., vol. A310, 1991, p. 398.

[9] Y. Nagai and T. Hyodo, "Position sensitive scintillation detector for $\gamma$-rays using a GSO crystal“, Nucl. Instr. and Meth. in Physics Res., vol. A349, 1994, p. 285.

[10] R. Pani et al., "Multi-crystal YAP:Ce detector system for position sensitive measurements", Nucl. Instr. and Meth. in Physics Res., vol. A348, 1994, p. 551 .

[11] A. Bandettini et al., "Characterization of the response of a double side $\mu$-strip silicon detector to X-rays in the diagnostic range", IEEE Trans. Nucl. Sci., vol. 40, 1993, p. 983.

[12] B. Alfano et al., "Digital imaging in radiology : preliminary results obtained with a high spatial resolution 2D silicon detector", IEEE Trans. Nucl. Sci., vol. 40, 1993, p. 987.

[13] F. Anghinolfi et al.,"A 1006 element hybrid silicon pixel detector with strobed binary output", IEEE Trans. Nucl. Sci., NS-39, 1992, p. 654.

[14] E. H. M. Heijne et al., "First operation of a $72 \mathrm{k}$ element hybrid silicon micropattern pixel detector array“, Nucl. Instr. and Meth. in Physics Res., vol. A349, 1994, p. 138.

[15] C. D'Ambrosio et al., "Photon counting with a hybrid photomultiplier tube (HPMT)“, Nucl. Instr. and Meth. in Physics Res., vol. A338, 1994, p. 389. 
[16] C. D'Ambrosio et al., "Further results on photon counting with small diameter scintillating fibres“, Nucl. Instr. and Meth. in Physics Res., vol. A345, 1994, p. 279.

[17] S. Baccaro et al., "Scintillation properties of YAP:Ce“, Nucl. Instr. and Meth. in Physics Res., vol. A361, 1995, p. 209. 
Table 1 Properties of YAP(Ce) Crystals

Chemical formula

Dopant

Crystal structure

Density

Hardness

Refractive index

Light emission peak

Light decay (1/e)

Photon yield

Light output

(compared to $\mathrm{NaI}(\mathrm{Tl})$ )

Radiation length

Avg. K X-ray energy of YAP

K-fluorescence yield of YAP
$\mathrm{YAlO}_{3}$ (non hygroscopic)

Cerium (0.05 - 1.0 weight \%)

Rhombic (no cleavage)

$\mathrm{g} \mathrm{cm}^{-3}$

5.37

Mohs

8.6

1.95

$\mathrm{nm} \quad 365$

ns 27 (X-ray excitation [17])

$(\mathrm{keV})^{-1} \quad 10$

$\% \quad 36$

$\mathrm{cm}$

2.7

$\mathrm{keV}$

15.2 (Yttrium)

0.36 (Yttrium) 


\section{Figure Captions}

1. Experimental arrangement of the $\gamma$-source, the $5 \mathrm{~mm}$ thick lead collimator (phantom) and the ISPA-camera. The insert shows the magnified lead collimator with its five hole positions.

2. Working principle of the ISPA $\gamma$-camera. The main elements for the $\gamma$-detection are represented (figure not to scale).

3. Simplified block diagram for the readout of the ISPA-camera. The strobe is generated by an appropriate amplification, shaping and discrimination of the back-side signal of the detector chip (self-triggering mode of the ISPA-tube).

4. Analog signal height distributions taken from the ISPA silicon chip rear contact (see fig. 2), for the planar YAP (a) and the YAP matrix (b). The YAP array shows higher light attenuation compared with the plate. The photoelectron peaks are clearly visible. The upper and lower discriminator settings are indicated. They form the window which activates the strobed signal output of the ISPA-tube and starts the pixel readout. For both spectra, the pedestal position is at channel number 107, and the threshold of the multi-channel analyzer is set at channel number 225 .

5. Two-dimensional view of the ISPA-anode pixel readout taken with the planar YAP (a) and the YAP matrix (b). The line A drawn through the images of three collimator holes corresponds to the intensity profiles of fig. 7.

6. Three-dimensional view of the ISPA-anode pixel readout. a) : planar- and b) : matrixYAP. The five towers show the respective collimator holes (insert of Fig. 1) and the excellent contrast for both cases.

7. Intensity distributions taken along the lines A of Fig. 5a (planar YAP) and Fig. 5b (YAP array), showing the profile of three $0.25 \mathrm{~mm}$ holes. The FWHMs are indicated.

8. Distributions of the measured centre-of-gravity precisions for the YAP plate (dashed curve) and array (full curve). The most probable values are $0.21 \mathrm{~mm}$ and $0.08 \mathrm{~mm}$, and the averages are $0.3 \mathrm{~mm}$ and $0.11 \mathrm{~mm}$, respectively. The tails are due to the contributions of gamma events with low photoelectron numbers and edge effects due to the limited chip size (for the plate). 